\title{
Victimización del personal de la escuela hacia estudiantes referidos a proyectos de integración escolar
}

\author{
Boris Villalobos-Parada*, Verónica López, Marian Bilbao**, Claudia Carrasco Aguilar***, Paula \\ Ascorra, Macarena Morales, Juan Pablo Álvarez, Álvaro Ayala, Dayana Olavarría y \\ Sebastián Ortiz \\ Pontificia Universidad Católica de Valparaíso, Chile. \\ **Universidad de Santiago de Chile ***Universidad de Playa Ancha
}

\author{
(Received May 8, 2014; Accepted December 14, 2014)
}

RESUMEN: El presente estudio analiza y compara las diferencias entre estudiantes con y sin diagnóstico de NEE, en relación a haber vivido experiencias de victimización escolar por parte de sus pares y del personal de la escuela. El estudio se realizó en establecimientos que brindan educación básica con Programas de Integración Escolar (PIE), de una comuna de Chile. Se aplicó un cuestionario de victimización a una muestra de 5,527 estudiantes, de los cuales 77 participaban en PIE. Los resultados del análisis de Odds Ratio y regresión logística indican mayores niveles de victimización de pares y del personal de la escuela en los estudiantes que participan en PIE en todas las categorías recogidas, con una razón de probabilidad de 2 a 1 para victimización de pares, y de 3 a 1 para victimización del personal de la escuela. Si para la población general de estudiantes, la probabilidad de que informen algún tipo de victimización de pares aumenta 3 veces si es que informan algún tipo de victimización de adultos de la escuela, esta proporción aumenta a 18 a 20 veces para el subgrupo de estudiantes con NEE referidos a PIE. Estos hallazgos sugieren que los estudiantes en PIE se constituyen en una población de alto riesgo frente a situaciones de victimización en la escuela, tanto de adultos en la escuela, como de sus pares, siendo especialmente preocupante el papel protector que pueden ejercer los adultos de la escuela, si participan activamente en la victimización hacia estos estudiantes. Los resultados son discutidos en relación a la necesidad de focalizar la promoción de factores protectores de modo de favorecer el desarrollo de una comunidad que cuide y proteja a todos sus estudiantes.

Palabras clave: victimización, bullying, necesidades educativas especiales, integración escolar, profesores.

\section{Prevalence of victimization from school staff to students referred to school integration programs}

\footnotetext{
ABSTRACT: This study analyzes and compares the experience of school victimization by peers and school staff, between students with and without diagnosis of special educational (SEN). 5,527 elementary school students studying in public schools with School Integration Programs (SIP) in one city of Valparaíso, Chile, participated in this study. Of these, 77 were students with SEN partici- 
pating in the SIP. Odds-ration and logistic regression analyses revealed higher levels of peer and school staff victimization for the subgroup of students with SEN in SIP programs, as compared to their non-SEN peers. The odds ratio was 2 to 1 for peer victimization and 3 to 1 for school staff victimization. Whereas for the general population of students, the odds that a student might inform at least one type of peer victimization increased 3 times if that student informed some type of school staff victimization, the same odds increased 18 to 20 times for the SEN students in SIP. These results suggest that students with SEN are an at-risk population with respect to school victimization, both from their peers as well as from school staff. Of special concern is the protective role that school staff is able to provide, if they themselves actively participate in victimizing these students. The results are discussed considering the need to focus on promoting protective factors that may favor the development of a caring and protective community for all students.

Key words: victimization, bullying, special educational needs, school integration, teachers.

\section{INTRODUCCIÓN}

Actualmente en Chile, uno de cada diez estudiantes reporta haber sido víctima de bullying en su establecimiento educacional, y de ellos un $25 \%$ indica vivirlo a diario (MINEDUC, 2012). Una revisión de literatura internacional indica que los estudiantes con NEE tienen el doble de probabilidades de estar involucrados como víctimas o perpetradores en casos de bullying, respecto de los estudiantes sin NEE (Rose, Monda-Amaya, \& Espelage, 2011). Este hecho requiere particular atención si se considera el aumento progresivo de las matriculas de estudiantes con NEE en las escuelas chilenas.

Desde 1990 a la fecha, el sistema educativo Chileno ha desarrollado una política para promover la participación de estudiantes con discapacidad y luego con necesidades educativas especiales (en adelante NEE). Esta política se materializa en los Proyectos de Integración Escolar (PIE) (Godoy, Meza, \& Salazar, 2004). En dichos proyectos, los estudiantes que presentan NEE, reciben apoyos focalizados en la forma de recursos educativos, atención de personal especialista y soportes especialmente diseñados (Comisión de Expertos de Educación Especial, 2004).

Para acceder a estos recursos los estudiantes deben ser diagnosticados con alguna de las categorías definidas actualmente en el Decreto 170, el cual regula los requisitos, los instrumentos, las pruebas diagnósticas y el perfil de los y las profesionales competentes que deberán aplicarlas, a fin de identificar a los alumnos con NEE. Una vez que los estudiantes son diagnosticados con NEE sus establecimientos educacionales reciben un monto adicional de subvención por cada uno de ellos, llamada subvención de educación especial (Peña, 2013). Esta subvención opera en base a la lógica de financiamiento del sistema educativo en Chile: el voucher o subvención a la demanda (Assael, Cornejo, González, 
Redondo, \& Sobarzo 2011; Bellei, 2013).

$\mathrm{Al}$ año 2010 el número de estudiantes en establecimientos con Proyectos de Integración Escolar (PIE), alcanzaba los 75,683 distribuidos en 4,110 establecimientos en distintos niveles en todo el país (MINEDUC, 2011). Tres años después de la implementación del Decreto 170, que incluyó a las NEE transitorias como parte de la subvención adicional, el número de estudiantes aumentó en un $200 \%$, con un total de 21,0331 estudiantes en 4,626 establecimientos educacionales municipales y particulares subvencionados en el año 2013 (SEGPRES, 2014). Particularmente explosivo fue el diagnóstico de estudiantes con Trastorno de Déficit Atencional, el que reportó un incremento promedio de $430 \%$ entre el año 2011 y el 2012 (OPECH, 2014) (ver Tabla 1).

Tabla 1. Variación del Diagnóstico de Necesidades Educativas Especiales para Subvención Adicional Asociada al Programa de Integración Escolar, entre el Año 2011 y 2012

\begin{tabular}{cccc}
\hline Región de Chile & $\mathbf{2 0 1 1}$ & $\mathbf{2 0 1 2}$ & Variación \\
\hline $\mathbf{1}$ & 150 & 516 & $344 \%$ \\
\hline $\mathbf{2}$ & 68 & 389 & $572 \%$ \\
\hline $\mathbf{3}$ & 33 & 106 & $321 \%$ \\
\hline $\mathbf{4}$ & 1024 & 2498 & $244 \%$ \\
\hline $\mathbf{5}$ & 858 & 3085 & $360 \%$ \\
\hline $\mathbf{6}$ & 471 & 1601 & $340 \%$ \\
\hline $\mathbf{7}$ & 288 & 1369 & $475 \%$ \\
\hline $\mathbf{8}$ & 1492 & 6001 & $402 \%$ \\
\hline $\mathbf{9}$ & 261 & 1399 & $536 \%$ \\
\hline $\mathbf{1 0}$ & 244 & 1146 & $470 \%$ \\
\hline $\mathbf{1 1}$ & 54 & 261 & $483 \%$ \\
\hline $\mathbf{1 2}$ & 123 & 513 & $417 \%$ \\
\hline $\mathbf{1 3}$ & 2723 & 8339 & $306 \%$ \\
\hline $\mathbf{1 4}$ & 62 & 411 & $663 \%$ \\
\hline $\mathbf{1 5}$ & 89 & 461 & $518 \%$ \\
\hline Promedio & & & $430 \%$ \\
\hline
\end{tabular}

Fuente: Adaptado de Observatorio de Políticas Educativas (OPECH) 2013.

Si bien la política de integración en Chile ha presentado avances sistemáticos para favorecer la participación de estudiantes con discapacidad en la escuela regular, incrementando su presencia en las escuelas, el proceso no ha estado exento de dificultades. En relación a ello, el informe del Estudio a Nivel Muestral de la 
Calidad del Proceso de Integración Educativa (CEAS, 2004) señala como limitaciones: la toma de decisiones centralizada respecto del uso de los recursos por parte de los sostenedores, quienes los focalizan en la contratación de personal por sobre la adquisición de materiales pedagógicos; el funcionamiento paralelo entre el PIE y el proyecto educativo institucional, donde el primero suele desarrollarse como un proyecto paralelo en la escuela; el predominio de un enfoque clínico y centrado en el diagnóstico, lo que se superpone a un abordaje pedagógico/social siendo los docentes de aula regular quienes mayormente reciben esta información; la falta de trabajo en equipo entre los docentes regulares y diferenciales, con claras dificultades para los docentes especialistas para prestar apoyos dentro del aula, lo que conlleva un trabajo de apoyo preferente en el aula de recursos; actitudes por parte de los docentes regulares, quienes tienden a responsabilizar al docente especialista del proceso de enseñanza de los estudiantes con NEE; y finalmente, la coexistencia de varias visiones sobre qué es la integración escolar, lo que lleva a diversas formas de abordar el trabajo en este contexto.

Por su parte, en el estudio llevado a cabo por UNICEF (2001) se exponen ciertas limitaciones presentes en la implementación de la integración escolar en nuestro país, que se alinean con las indicadas en el estudio anterior. Cabe resaltar entre otras: la falta de articulación dentro del sistema escolar entre financiamiento, currículo y formación docente; el planteamiento desde una perspectiva clínica y no desde un enfoque pedagógico y social de la integración; la falta de continuidad y articulación entre los distintos niveles (preescolar, básica y media); la falta de garantías de participación en los establecimientos con PIE para los estudiantes con NEE; una baja participación de las familias y la comunidad educativa en la formulación de los proyectos; y finalmente, la falta de formación inicial de los docentes focalizada en la diversidad.

En el año 2013, es decir, cuatro años después de la promulgación del decreto 170, Fundación Chile entrega un informe respecto de la implementación de los PIE con foco en los proyectos para estudiantes con NEE transitorias. El informe concluye que la implementación de los sistemas de diagnóstico y contratación de profesionales ha sido exitosa, favoreciendo la identificación de las NEE de los estudiantes. No obstante señala, en línea con los documentos precedentes, que la implementación ha sido difícil dentro de las escuelas para lograr el aprendizaje de los estudiantes, faltando para ello: el trabajo coordinado entre profesionales, la institucionalización de los PIE el interior de las escuelas, la capacitación del personal y priorización de los PIE, y la disponibilidad de recursos para la implementación (Marfán, Castillo, González, \& Ferreira, 2013).

En el año 2014, López et al. presentaron un análisis de las concepciones y prácticas sobre la integración desde la perspectiva del Decreto 01/98. Para ello realizan un análisis del discurso de esta política educativa y de entrevistas y observaciones no participantes a diversos actores relacionados con su implementación en escuelas de la Región de Valparaíso en Chile. En el estudio se destaca el carácter híbrido de la política de integración la que promueve un ideario de inclusión, pero que termina por conducir a una implementación desde un modelo 
médico, empleando medidas focalizadas en los estudiantes diagnosticados con NEE. Esta lógica se impone en el trabajo de la escuela, y dificulta la implementación de una perspectiva de plena inclusión para los estudiantes. Esto se hace más evidente dada la relevancia que adquiere el etiquetamiento de algunos de ellos como los niños PIE, asociando además su diagnóstico y etiqueta a la entrega de una subvención especial (López, Julio, Morales, Rojas, \& Pérez, 2014).

Peña (2013) analizó discursivamente el Decreto 170, que regula actualmente la asignación de los recursos de subvención de educación especial. La autora concluye que este decreto establece una perspectiva médica para la categorización de los estudiantes, indicando quién, cómo y con qué instrumentos debe hacerla. Esta perspectiva médica se conecta con una política neoliberal de subvención a la demanda, mediante la cual los recursos especiales quedan sujetos al diagnóstico que un especialista realice en un establecimiento educacional (Peña, 2013). Estos medios de diagnóstico condicionan el acceso a medios preferenciales de acceso, participación y promoción, mediante la subvención escolar especial. De esta manera, la subvención especial que supone el financiamiento de profesionales especialistas, recursos adaptados y medios de acceso del programa de educación especial (MINEDUC, 2011), quedando condicionada al diagnóstico del profesional idóneo que el decreto determine, con los medios que el decreto exija y con las categorías que éste delimite.

De esta manera, el encuentro de la subvención especial y el diagnóstico como medidas focalizadas y de excepción, descritas previamente en estos análisis (López et al., 2014; Peña, 2013) dan cuenta de un mecanismo de provisión de recursos, centrado en la noción de capacidad de los estudiantes para aprender de forma normal o en la necesidad de normalizarlos para que aprendan. Ello puede vincularse con lo que Campbell (2009) denomina aptismo (ableism, en inglés). Este concepto se entiende como una actitud que devalúa o diferencia la discapacidad a través de la valoración de la aptitud corporal equiparada a la normalidad. Así, lo que se contrasta es la condición particular de un estudiante determinado en función de su capacidad para responder a las demandas del contexto educativo. Esto resalta la concepción de las NEE como una situación individual que debe ser identificada diagnósticamente por especialistas, y luego ser gestionada por los establecimientos con los recursos que la subvención dispone relevando el foco en el diagnóstico y el señalamiento de la condición particular de los sujetos (Peña, 2013; Soldatic \& Meekosha, 2012).

En el contexto educativo chileno, la participación en la escuela regular de los estudiantes con NEE está regulada por la Ley de Inclusión de Personas con Discapacidad (Ley 20,422) y el Decreto 170 de Educación Especial (MINEDUC, 2009). Si bien el principio que sostiene la Ley 20,422 es el de la inclusión, en la práctica las escuelas operan con los mecanismos derivados del decreto 170, el cual deriva directamente de la ley de subvenciones, promulgado en el año 2009, es decir, prácticas orientadas desde la integración. Esto supone una contradicción que puede ser entendida como hibridez paradigmática (García Canclini, 1990). Como señalan López et al., 2014 (p.14): "las sociedades latinoamericanas tien- 
den a procesos de hibridación frente a las demandas de globalización cultural. La política educativa de integración social, entendida como práctica social, presenta características de hibridez y procesos de hibridación en tanto justifica acciones de integración desde un enfoque de inclusión, que imposibilitan o, a lo menos, dificultan la misma inclusión."

El problema de investigación bajo el cual se enmarca este estudio, son los efectos de este tipo de políticas de integración escolar. Como señala la literatura, uno de los efectos de la subvención adicional a la demanda asociada al diagnóstico clínico individual, es el etiquetamiento y la segregación escolar (López et al., 2014; Soldatic \& Meekosha, 2012; Peña, 2013). Adicionalmente, nos preguntamos si acaso este tipo de políticas, que enmarcan un cierto tipo de participación académica y social de los estudiantes, tienen también efectos sobre la vida social de los estudiantes, particularmente, sobre sus experiencias de bullying o victimización escolar por parte del profesorado y personal de la escuela.

\section{VICTIMIZACIÓN DE ESTUDIANTES DESDE EL PERSONAL DE LA ESCUELA}

En la revisión bibliográfica realizada en las bases de datos Scopus, Scielo, Redalyc, y Google Scholar durante el tercer trimestre del año 2014, utilizando como palabras claves "school violence", "bullying", "teachers", y "special educational needs" (y sus equivalentes en español), no se encontraron estudios específicamente referido a la victimización de personal de la escuela hacia los estudiantes referidos con NEE. Dado este antecedente se revisan investigaciones referidas a la victimización de estudiantes en general por parte del personal de la escuela.

Cabe señalar que esta literatura es también escasa. La mayor parte de los estudios empíricos que consideran a los profesores y otros integrantes del personal de la escuela (school staff) preguntan a éstos su percepción sobre el nivel de violencia entre estudiantes (Bradshaw, Sawyer, \& O'Brennan, 2007) o bien, la violencia o victimización que ellos reciben por parte de los estudiantes (Shields, Nadasen, \& Hanneke, 2015). Son pocos los estudios que preguntan a los estudiantes por el nivel de victimización que reciben por parte de sus profesores o personal del centro escolar.

En el año 2002, Benbenishty, Zeira, y Astor, condujeron una investigación en Israel con 10,410 estudiantes. Un $24.9 \%$ de los estudiantes reporta agresiones emocionales (verbales); mientras que un $18.7 \%$ reporta agresiones físicas de parte del personal de la escuela (school staff). Finalmente un $18.2 \%$ de los estudiantes indica haber sufrido alguna agresión de índole sexual por parte del personal de la escuela. Los grupos más vulnerables a estas situaciones de violencia son los hombres, de educación secundaria, de escuelas árabes, con una alta concentración de estudiantes provenientes de familias con bajos ingresos y bajos niveles educativos (Benbenishty et al., 2002).

En línea similar y también en Israel, Khoury-Kassabri (2006), encontró que 
los estudiantes reportan más agresiones de índole verbal-emocional de parte del personal de la escuela, específicamente, sus profesores. Así, reportan un 30,9\% en primaria, $35 \%$ en pre-secundaria, y un $34,5 \%$ en secundaria. En cuanto a la agresión física, los estudiantes $25 \%$ en pre-secundaria y $18 \%$ en secundaria. Referido a la victimización sexual, un cuarto de los estudiantes en pre- secundaria y un $8 \%$ en secundaria, señalan haberla vivido en sus escuelas. Así mismo, los estudiantes más vulnerables a estas situaciones son los hombres, árabes y que estudian en escuelas de vecindarios de bajo nivel socioeconómico y donde asisten estudiantes cuyas familias son señaladas con bajo nivel socioeconómico. Así mismo, la autora concluye que estas situaciones pueden estar más relacionadas con factores de organización de la escuela en sus distintos niveles, primaria, presecundaria y secundaria; en los dos primeros los estudiantes reportan más victimización física. La misma autora (Khoury-Kassabri, 2009), encontró que los estudiantes identificados como bully-victima reportan niveles significativamente más altos de agresión del personal de la escuela que los estudiantes señalados como bullys, victimas o no involucrados en estas situaciones. Nuevamente los hombres señalan significativamente más agresiones que las mujeres.

En España, Lucas-Molina, Williamson, Pulido y Pérez-Albéniz (2015) realizaron un estudio multinivel para analiar los efectos de las relaciones entre estudiantes y profesores, sobre la victimización de pares. Sus resultados indican que tanto la agresión de profesores a estudiantes, como la agresión de estudiantes a profesores, se asocian un incremento de la victimización de pares. De manera inversa, el apoyo de profesores se asoció a una disminución de victimización de pares.

Datos recientes publicados por nuestro equipo, dan cuenta de que en el contexto chileno los estudiantes referidos con NEE en las escuelas regulares son un grupo de riesgo frente a situaciones de victimización por parte de sus pares. En términos generales, los estudiantes referidos con NEE reportan 2.45 agresiones de tipo sexual, 2.42 agresiones a través de medios digitales, 2.4 agresiones mediante exclusión social, 2.14 agresiones físicas y 1.55 agresiones por verbales por cada agresión específica que reportan sus pares que no han sido referidos con NEE (Villalobos-Parada et al., 2014); es decir, en la mayoría de los casos los estudiantes referidos con NEE reportan más del doble de agresiones que los estudiantes que no participan de los PIE. Los datos precedentes sugieren que los estudiantes referidos con NEE, están más expuestos que sus pares a sufrir agresiones por parte de personal de sus escuelas, lo cual se asocia a victimización por parte de sus pares.

Es importante señalar que investigaciones recientes señalan que uno de los mejores predictores de la violencia entre pares, es la presencia de victimización verbal por parte de los docentes (López, Bilbao, \& Rodríguez, 2011). De este modo, podemos inferir que donde existe victimización del profesorado, personal administrativo u otros adultos en la escuela, es probable que exista alguna forma de agresión a los estudiantes por parte de sus pares.

De este modo, los antecedentes dan cuenta de que es posible que los estudian- 
tes referidos con NEE estén en riesgo de victimización por parte de los adultos en la escuela. Esto considerando la relación que existe entre victimización verbal del profesor y violencia entre pares. Asimismo, bajo nivel socioeconómico se relaciona con la victimización por parte de los adultos en la escuela, y es en dicho contexto donde se concentran la mayor cantidad de PIE en Chile. Es importante entonces indagar sobre la prevalencia de victimización por parte del personal de la escuela hacia los estudiantes referidos con NEE que asisten a los PIE en las escuelas regulares.

Así, este estudio se planteó dos hipótesis. Primero, que dado el alto nivel de victimización que se ha detectado por parte de los pares, se encontrarán mayores niveles de victimización por parte de persona del establecimiento. Segundo, que la victimización del personal de la escuela predice la victimización de pares, y que esta asociación es aún más fuerte para el subgrupo de estudiantes con NEE.

\section{MÉTODO}

\section{Participantes}

Participaron 5,527 estudiantes de 44 escuelas municipales de una comuna de la región de Valparaíso, Chile, que cursan desde $4^{\circ}$ a $8^{\circ}$ año de enseñanza básica. De ellos, 77 estudiantes están referidos al PIE, mientras que 5,450 no lo están en PIE. De estos estudiantes $51.5 \%$ son varones y $48.5 \%$ mujeres. Las edades variaron desde los 8 a los 18 años de edad, con un promedio de 11.7 años de edad (DS = 1.67).

La información respecto de la participación en los PIE de cada estudiante se obtuvo cruzando los datos de la encuesta con las bases de datos del Ministerio de Educación. Luego este dato fue añadido a la información recogida en este estudio, creando una nueva variable que permitió la comparación de los grupos.

\section{Instrumentos}

Los instrumentos empleados en este estudio son parte de la batería de cuestionarios de la encuesta "La Convivencia en mi Escuela" cuyas propiedades psicométricas se encuentran publicadas en López, Ascorra, Bilbao, Carrasco, Morales, Villalobos y Ayala (2013). Esta batería de encuestas fue creada y validada en el marco del proyecto FONDEF-IDEA CA12i10243 "Diseño, desarrollo y validación de un sistema de monitoreo de la convivencia escolar". Todos los instrumentos cuentan con la opción "No sé/No quiero contestar" para evitar forzar la respuesta de los estudiantes. La batería de cuestionarios, está compuesta por escalas de clima escolar, victimización de estudiantes, victimización de personal del establecimiento, intimidación a profesores, peligrosidad en la escuela, contribución a la escuela y satisfacción con la vida. Para efectos de los análisis acá presentados, se emplearon las escalas "Victimización de Personal del Establecimiento" y "Victimización de Pares". 


\section{Victimización de Profesores y Personal de la Escuela:}

Esta escala se compone de 4 ítems $(\alpha=.91)$ que consulta por la frecuencia de ocurrencia de hechos violentos perpetrados en el último mes realizados por un profesor o personal del establecimiento. Tiene un rango de respuesta de tres puntos: "Nunca en este mes" (1), "Una o dos veces en este mes" (2) y "Tres o más veces en este mes" (3). Esta variable fue convertida a una variable dicotómica, "Ocurrió" (1), y "No Ocurrió" (0). De este modo se consideró la suma de hechos señalados, con la posibilidad de haber sufrido hasta 4 actos distintos de violencia por parte de pares en el último mes. La comprende cuatro tipos de agresiones específicas que los estudiantes podían reportar por parte del personal, siendo estas: Agresión Verbal (Un adulto de la escuela te insultó, humilló o se burló de ti); Agresión física (Un adulto de la escuela te zamarreó o te empujó a propósito; Un adulto de la escuela te pellizcó o te cacheteó) y Acoso sexual (Un adulto de la escuela te tocó o intentó tocarte de manera sexual). Los resultados del análisis factorial exploratorio (rotación Varimax) indicaron que la escala se comporta de manera unifactorial.

\section{Victimización entre Estudiantes:}

Esta escala se compone de 27 ítems $(\alpha=0.94)$ que consulta por la frecuencia de ocurrencia de hechos violentos perpetrados en el último mes realizados por un compañero o par. Tiene un rango de respuesta de tres puntos: "Nunca en este mes" (1), "Una o dos veces en este mes" (2) y "Tres o más veces en este mes" (3). Esta variable fue convertida a una variable dicotómica, "Ocurrió" (1), y "No Ocurrió" (0). De este modo se consideró la suma de hechos señalados, con la posibilidad de haber sufrido hasta 27 actos distintos de violencia por parte de pares en el último mes. Los resultados del análisis factorial exploratorio indicaron que la escala es multifactorial y se descompone en cinco dimensiones: Victimización Verbal $(\alpha=.80)$, Victimización Física $(\alpha=.86)$, Victimización a través de medios digitales, o ciber-victimización $(\alpha=.83)$, Victimización Social o Exclusión social intencionada $(\alpha=.74)$, y Victimización de índole sexual $(\alpha=.89)$.

\section{Análisis de Datos}

Los puntajes fueron convertidos a una variable dicotómica, "Ocurrió" (1), y "No Ocurrió" (0). El análisis de probabilidad se hizo mediante el uso de tablas de contingencias de 2 x 2. Para ello se usaron las categorías "En PIE / No en PIE" y "Con Victimización / Sin Victimización". Considerando que se trata de un estudio censal de los estudiantes en escuela públicas, se realizó un análisis de riesgo para la victimización con la estimación de razón de posibilidad (Odds Ratio). El índice Odds-Ratio permite estimar la proporción de ocurrencia de un evento para un grupo, en comparación a otro. Estos análisis se realizaron utilizando el paquete estadístico SPSS 18. 


\section{RESULTADOS}

Respecto a la agresión por parte del personal del establecimiento, la Figura 1 muestra que los estudiantes referidos a los PIE muestran más agresiones que sus pares en el último mes en términos generales y en cada una de las categorías consultadas. Es decir, los estudiantes en los PIE reportan más agresiones sexuales, golpes, empujones y violencia verbal que sus pares, duplicando incluso el porcentaje de reportes respecto de sus pares en todas las categorías.

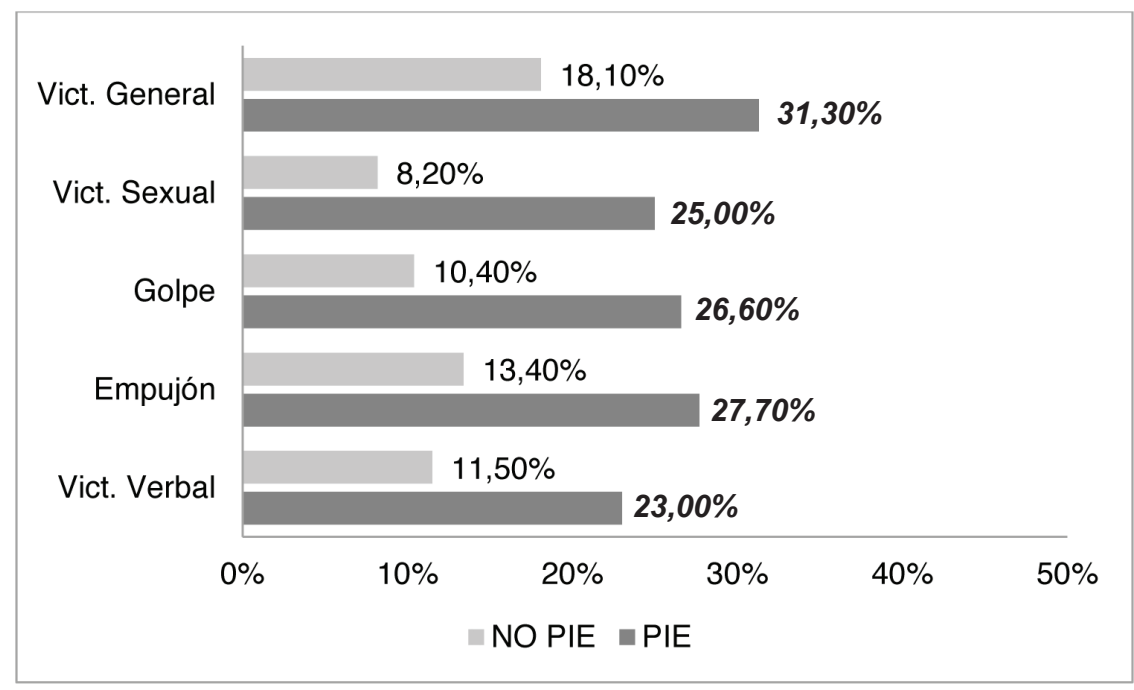

Figura 1. Victimización del personal del establecimiento hacia estudiantes en PIE

Por otra parte, con respecto a la agresión por parte de los pares (otro estudiante del centro escolar), la Figura 2 muestra que los estudiantes referidos a los PIE muestran también más agresiones que sus pares en el último mes en todas las dimensiones analizadas, duplicando las frecuencias solo en el caso de victimización vía ciberbullying y victimización sexual. 


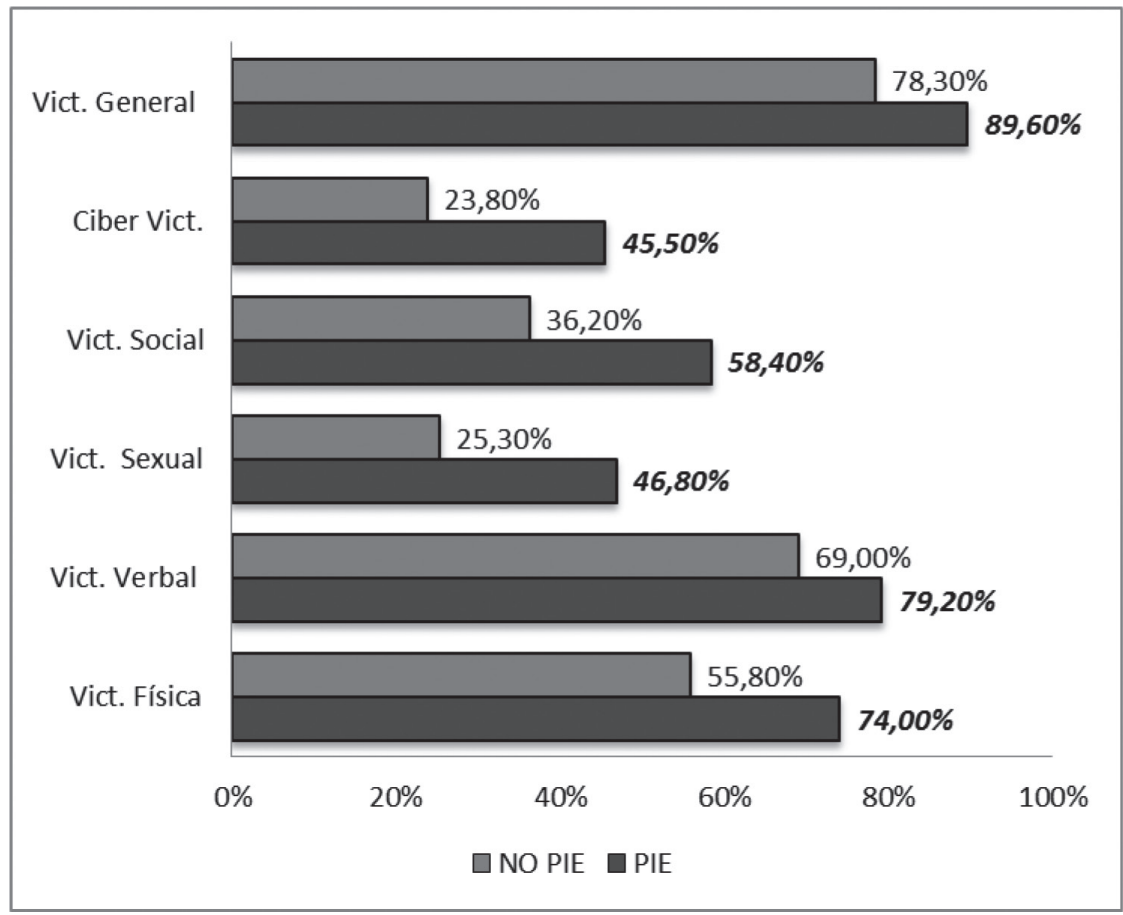

Figura 2. Victimización de estudiantes en PIE y estudiantes que no están en PIE.

Nota: estos datos han sido informados en Villalobos-Parada et al., 2014.

La Tabla 2 resume los porcentajes, número de estudiantes y razones de probabilidad para cada tipo de victimización de parte del personal de la escuela hacia los estudiantes en PIE. Los resultados del análisis de odds ratio indican que por cada agresión que reportan los estudiantes que no participan del PIE de su establecimiento, los estudiantes en el PIE reportan 3.71 agresiones sexuales, 3.12 golpes, 2.47 empujones y 2.3 agresiones verbales por parte del personal del establecimiento. Para la escala general de victimización del personal, los estudiantes en PIE reportan 2.07 agresiones por cada agresión que reportan sus compañeros.

La Tabla 3 presenta los porcentajes, número de estudiantes y razones de probabilidad para cada tipo de victimización de parte de los compañeros del centro escolar hacia los estudiantes en PIE. Por cada agresión que reportan los estudiantes que no participan del PIE de su establecimiento, los estudiantes en el PIE reportan 1.95 agresiones para la escala general. El desglose por tipo de victimización varía entre 1.55 para victimización verbal y 2.45 para victimización sexual.

En síntesis, los resultados del análisis descriptivo y de odds ratio sugieren que los estudiantes con NEE referidos a PIE presentan un riesgo relativo mayor de ser víctimas de agresiones tanto por parte de sus compañeros, como por parte del profesorado y personal del centro escolar. Los hallazgos indican que el riesgo 
relativo es mayor para la victimización por parte de los adultos de la escuela (OR $=2.07)$ que por parte de los pares $(\mathrm{OR}=1.95)$. Especialmente preocupante es el mayor riesgo de ser victimizados por el personal de manera sexual $(\mathrm{OR}=3.71)$ y por la vía de golpes físicos $(\mathrm{OR}=3.12)$.

Tabla 2. Riesgo Relativo de Victimización del Personal del Establecimiento a Estudiantes en Proyectos de Integración Escolar $(N=5.527)$

\begin{tabular}{ccccccc}
\hline & \multicolumn{2}{c}{ Estudiantes en PIE } & \multicolumn{2}{c}{$\begin{array}{c}\text { Estudiantes no en } \\
\text { PIE }\end{array}$} & $\begin{array}{c}\text { Razón de } \\
\text { posibilidad } \\
\text { (Odds Ratio) }\end{array}$ & $\begin{array}{c}\text { Intervalo de } \\
\text { Confianza } \\
(95 \%)\end{array}$ \\
\hline Victimización & $\mathrm{n}$ & $\%$ & $\mathrm{n}$ & $\%$ & & \\
\hline General & 21 & 31.3 & 915 & 18.1 & 2.07 & $(1.23-3.49)$ \\
Sexual & 16 & 25 & 403 & 8.2 & 3.71 & $(2.09-6.6)$ \\
Golpe & 17 & 26.6 & 512 & 10.4 & 3.12 & $(1.77-5.48)$ \\
Empujón & 18 & 27.7 & 664 & 13.4 & 2.47 & $(1.43-4.28)$ \\
Verbal & 14 & 23 & 566 & 11.5 & 2.3 & $(1.26-4.21)$
\end{tabular}

*OR= indicador que sugiere la proporción entre dos eventos. De tener un valor de 1 , indica una proporción $1: 1$.

Tabla 3. Riesgo Relativo de Victimazación entre Pares para Estudiantes en Pie $(n=81)$ y Estudiantes que No Están en PIE $(N=5.527)$

\begin{tabular}{|c|c|c|c|c|c|c|}
\hline \multirow[b]{2}{*}{ Victimización } & \multicolumn{2}{|c|}{ Estudiantes en PIE } & \multicolumn{2}{|c|}{$\begin{array}{l}\text { Estudiantes no en } \\
\text { PIE }\end{array}$} & $\begin{array}{l}\text { Razón de } \\
\text { posibilidad } \\
\text { (Odds } \\
\text { Ratio)* }\end{array}$ & \multirow{2}{*}{$\begin{array}{l}\text { Intervalo de } \\
\text { Confianza } \\
(95 \%)\end{array}$} \\
\hline & $\mathrm{n}$ & $\%$ & $\mathrm{~N}$ & $\%$ & & \\
\hline General & 71 & 87.75 & 4465 & 79.57 & 1.95 & $(1.00-3.78)$ \\
\hline Ciber & 35 & 43.21 & 1358 & 23.87 & 2.42 & $(1.56-3.78)$ \\
\hline Social & 47 & 58.02 & 2079 & 36.50 & 2.40 & $(1.54-3.75)$ \\
\hline Sexual & 37 & 45.68 & 1453 & 25.55 & 2.45 & $(1.57-3.81)$ \\
\hline Verbal & 63 & 77.78 & 3942 & 69.30 & 1.55 & $(.91-2.62)$ \\
\hline Física & 59 & 72.84 & 3165 & 55.64 & 2.14 & $(1.30-3.50)$ \\
\hline
\end{tabular}

\footnotetext{
*OR $=$ indicador que sugiere la proporción entre dos eventos. De tener un valor de 1, indica una proporción $1: 1$.
} 
Nota: estos datos han sido informados en Villalobos-Parada et al., 2014.

Para responder a la pregunta en torno al papel que cumple la victimización del profesorado en la victimización de pares, realizamos un análisis de regresión logística con la victimización del personal como variable predictora. La hipótesis fue que la victimización del personal de la escuela predice la victimización entre pares, tanto para la población general de estudiantes, como para el subgrupo de estudiantes con NEE referidos a PIE.

La Tabla 4 muestra los resultados para la población general de estudiantes. Para aquellos estudiantes que informan algún tipo de victimización por parte del personal de la escuela, la probabilidad de que informen, además, algún tipo de victimización por parte de sus pares es de $3.43(\mathrm{p}<.001)$. Es decir, la probabilidad de que un estudiante señale ser agredido por sus pares aumenta 3 veces, si este estudiante reporta ser victimizado por adultos en la escuela.

Tabla 4. Resumen del Análisis de Regresión Logística Prediciendo la Victimización Entrepares

\begin{tabular}{ccccccc}
\hline Variable & $\beta$ & E.S & $\begin{array}{c}\text { Odds } \\
\text { Ratio }\end{array}$ & 95\% IC & Wald & $p$ \\
\hline $\begin{array}{c}\text { Victimización } \\
\text { de Personal de } \\
\text { la escuela }\end{array}$ & 1.21 & .12 & 3.36 & {$[2.66-4.23]$} & 104.2 & .000 \\
\hline
\end{tabular}

La Tabla 5 muestra los resultados para los estudiantes en PIE. Como se puede observar, en esta primera ecuación de regresión logística realizada, la victimización del personal de la escuela no resultó un predictor de la victimización que reportan por parte de sus pares $(p=.998)$, aun cuando el índice Odds Ration mostró un valor de 3.36. Sin embargo, al analizar la tabla de contingencia entre las variables del estudio (ver Tabla 6), observamos que en el subgrupo de 77 estudiantes referidos a los PIE, 8 (11.94\%) no reportan victimización por parte de ninguno de los autores consultados. Del resto, $38(56.72 \%)$ reportan agresiones por parte de sus pares, pero no del personal de la escuela. Finalmente, 21 (31.34\%) de los estudiantes referidos a los PIE reportan victimizaciones de ambos actores, personal de la escuela y sus pares. Los tamaños de los subgrupos que se producen en la tabla de contingencia no permiten estimar el modelo, ya que el grupo de interés es inferior a 30 casos. Así mismo, el alto nivel de agresiones totales que reportan los estudiantes en PIE (59 casos, correspondiente al 77\%), puede dificultar que el modelo pueda discriminar la relación entre las variables. 
Tabla 5. Resumen del Análisis de Regresión Logística Prediciendo la Victimización entre Pares (Estudiantes en PIE)

\begin{tabular}{lllllll}
\hline Variable & $\beta$ & E.S & $\begin{array}{l}\text { Odds } \\
\text { Ratio }\end{array}$ & $95 \%$ IC & Wald & $p$ \\
\hline $\begin{array}{l}\text { Victimización } \\
\text { de Personal de } \\
\text { la escuela }\end{array}$ & 19.65 & 8770.83 & $3.40^{8}$ & {$[.000]$} & .000 & .998 \\
\hline
\end{tabular}

Tabla 6. Relaciones de Contigencia Victimización de Personal y Victimización de Pares

\begin{tabular}{llccr}
\hline & & \multicolumn{2}{c}{ Victimización de Pares } & \multirow{2}{*}{ Total } \\
\cline { 3 - 4 } Victimización de & Sin Agresión & 8 & \\
\cline { 3 - 4 } Personal & Agresión & 0 & 38 & 46 \\
Total & & 8 & 21 & 21 \\
& & & 59 & 67 \\
\hline
\end{tabular}

Por estos motivos, decidimos incorporar la técnica de bootstrapping (Field, 2009) para poder obtener los intervalos de confianza y estimaciones necesarias para establecer la relación entre las variables. Esta técnica permite generar varias muestras desde los datos, para permitir estimar los estadísticos de interés de cada muestra extraída, como si los datos fuesen cercanos a la población. La Tabla 7 muestra los resultados del análisis empleando la técnica de bootstrap. El análisis indica que para los estudiantes referidos a los PIE que informan victimización por parte de adultos en escuela, la probabilidad de que un estudiante en PIE reporte victimización por parte de sus pares es 18 a 20 veces mayor, si es que reporta victimización por parte del personal la escuela.

Tabla 7. Resumen del Análisis de Regresión Logística con Bootstrapping Prediciendo la Victimización entre Pares para el Subgrupo de Estudiantes en PIE

\begin{tabular}{llllll}
\hline Variable & B & Sesgo & E.S & $95 \%$ IC & $p$ \\
\hline Victimizació & & & & & \\
$\mathrm{n}$ de & \multirow{2}{*}{19.65} & -.040 & .461 & {$[18.512-$} & .001 \\
$\begin{array}{l}\text { Personal de } \\
\text { la escuela }\end{array}$ & & & & $20.366]$ & \\
\hline
\end{tabular}




\section{CONCLUSIONES}

Los resultados entregan apoyo a las dos hipótesis de este estudio: primero que, que el nivel de victimización reportado por los estudiantes referidos a los PIE es sustancialmente mayor que el reportado por sus compañeros no referidos a dichos proyectos; $y$ segundo, que el riesgo de ser victimizado por sus pares aumenta cuando recibe victimización por parte de los adultos de la escuela, siendo este riesgo aún más alto para los estudiantes referidos a PIE.

Estos hallazgos sugieren que los estudiantes en PIE se constituyen en una población de alto riesgo frente a situaciones de victimización en la escuela, tanto de adultos en la escuela, como de sus pares.

Así, mientras la razón de agresiones de pares reportadas entre estudiantes no referidos y referidos con NEE es de 2 a 1 en promedio; la razón de agresiones de docentes y personal de la escuela es de 3 a 1 en promedio. Es decir, que por cada agresión que indican sufrir los estudiantes que no han sido referidos con NEE, los estudiantes referidos a los PIE reportan tres.

Por otra parte, si para la población general de estudiantes, la probabilidad de que informen algún tipo de victimización de pares aumenta 3 veces si es que informan algún tipo de victimización de adultos de la escuela, esta proporción aumenta a 18 a 20 veces para el subgrupo de estudiantes con NEE referidos a PIE.

Existe evidencia que vincula la agresión verbal del docente a la violencia entre pares (López et al., 2011), siendo la conducta del docente un predictor de la violencia. Esto es de particular gravedad considerando que para los estudiantes en situaciones de victimización de pares el apoyo social de los docentes y adultos en la escuela se vuelve de suma importancia (Flaspohler, Elfstrom, Vanderzee, Sink, \& Birchmeier, 2009). Así, considerando el alto riesgo al que están expuestos los estudiantes referidos a los PIE, el hecho de que el nivel de agresiones reportadas sea mayor en el caso de los adultos en la escuela genera una debilidad en los apoyos disponibles para los estudiantes.

Cabe señalar que nuestros datos fueron recogidos en el contexto de educación municipal en una comuna de la Región de Valparaíso. En Chile la segregación del sistema educativo produce que los estudiantes más pobres se concentren en el sistema público administrado por las municipalidades (Bellei, 2013; Cornejo, 2006). La evidencia internacional (Benbenishty et al., 2002; Khoury-Kassabri, 2006) indica una asociación entre alta victimización y bajo nivel socioeconómico.

Así, considerando que la concentración de niños de bajo nivel socioeconómico es un factor de riesgo, la condición de haber sido referido al PIE se suma en el contexto de la educación pública, sobre todo considerando que las escuelas municipales concentran la mayor cantidad de estudiantes en PIE (DIPRES, 2009).

Se ha cuestionado que en el actual contexto de integración escolar en Chile, con foco en el déficit, diagnóstico y tratamiento, las prácticas de integración puedan tener efectos negativos en la convivencia de los estudiantes en PIE (López et al., 2014; Peña, 2013; Villalobos-Parada, 2014). El presente análisis sugieren 
que debemos comenzar a abordar los efectos del etiquetamiento y la segregación que conlleva una política de atención a la diversidad bajo una modalidad de la integración escolar sujetada a las lógicas neoliberales de subvención a la demanda (Peña, 2013), que para el caso de la subvención adicional para NEE implica el diagnóstico y consecuente etiquetamiento y segregación del estudiante con NEE (López et al., 2014). Los resultados de este estudio permiten concluir que uno de estos efectos es la mayor probabilidad de ser victimizados tanto por sus pares, como por el profesorado y el personal de la escuela. De allí, que consideramos que estos resultados advierten sobre la necesidad de comprender los efectos sociales de las políticas de integración escolar de estudiantes con NEE en contextos de educación regular. Es importante, por lo tanto, desarrollar estudios que permitan establecer la relación entre las políticas de integración o inclusión y los fenómenos aquí descritos.

Asimismo, para las escuelas que optan por la atención a la diversidad, es importante incorporarla a la gestión integral del establecimiento. Como señalan los estudios sobre la implementación de la integración escolar en Chile (UNICEF, 2001; Ceas, 2004; Marfán et al., 2013), la atención de los estudiantes referidos con NEE ha sido delegada a los especialistas de los PIE, y no ha se ha incorporado a la estructura del establecimiento. Esto es insuficiente para la atención a la diversidad y la aspiración de una plena participación para los estudiantes (Blanco, 2009).

Los resultados de este estudio nos permiten concluir que la sola presencia de los estudiantes referidos con NEE en escuelas regulares no garantiza su plena integración o inclusión. Ello probablemente requiere que las escuelas articulen sus proyectos educativos en consideración a la creciente diversidad que en ellas se educa (Ainscow, 2012; Avramidis, Bayliss, \& Burden, 2000; Carrington, 1999).

Considerando los efectos negativos que la agresión de pares y el efecto mediador de adultos tiene sobre el bienestar de los estudiantes (Flaspohler et al., 2009) es imperativo trabajar con las escuelas en la promoción de estrategias positivas para la prevención de la agresión y la promoción de relaciones positivas.

Del mismo modo, es necesario formar y proveer al personal de la escuela con estrategias para atender y tratar con una diversidad creciente en las escuelas, de tal modo de disminuir potenciales conductas agresivas con los estudiantes.

Finalmente, respecto de las limitaciones de este estudio, reconocemos la necesidad de conducir análisis más exhaustivos que permitan, por ejemplo, generalizar sus resultados a la población. Instamos los investigadores interesados en este fenómeno, a conducir investigaciones de carácter cualitativo para comprender cómo opera y cómo afecta la victimización del profesorado y personal de la escuela, a los estudiantes con NEE.

\section{AGRADECIMIENTOS}

Este estudio fue financiado por el Proyecto FONDEF IT 14i10132. 


\section{REFERENCIAS}

Ainscow, M. (2012). Haciendo que las escuelas sean más inclusivas: lecciones a partir del análisis de la investigación internacional. Revista de Educación Inclusiva, 5(1), 39-49. Retrieved from http://dialnet.unirioja.es/descarga/articulo/4105297.pdf

Assael, J., Cornejo, R., González, J., Redondo, J. M., Sánchez, R., \& Sobarzo, M. (2011). La empresa educativa chilena. Educacao \& Sociedade, 32(115), 305-322. Retrieved from http://www.captura.uchile.cl/handle/2250/131899

Avramidis, E., Bayliss, P., \& Burden, R. (2000). A survey into mainstream teachers' attitudes towards the inclusion of children with special educational needs in the ordinary school in one local education authority. Educational psychology, 20(2), 191-211.

Bellei, C. (2013). El estudio de la segregación socioeconómica y académica de la educación chilena. Estudios Pedagógicos, XXXIX(1), 325-345.

Benbenishty, R., Zeira, A., \& Astor, R. A. (2002). Children's reports of emotional, physical and sexual maltreatment by educational staff in Israel. Child Abuse \& Neglect, 26(8), 763-782. doi:10.1016/S0145-2134(02)00350-2

Blanco, R. (2006). Hacia una escuela para todos y con todos. Boletín del Proyecto Principal de Educación para América Latina y el Caribe, 48, 55-72.

Bradshaw, C. P., Sawyer, A. L., \& O'Brennan, L. M. (2007). Bullying and peer victimization at school: Perceptual differences between students and school staff. School Psychology Review, 36(3), 361-382

Campbell, F. K. (2009). Contours of Ableism. Basingstoke: Palgrave Macmillan.

Carrington, S. (1999). Inclusion needs a different school culture. International JournalofInclusive Education, 3(3), 257-268. doi:10.1080/136031199285039

Ceas, L. (2004). Estudio a Nivel Muestral de la calidad del Proceso de Integración Educativa. Mineduc.

Comisión de Expertos de Educación Especial, M., Comisi, I. D. E. L. A., \& Expertos, D. E. (2004). NUEVA PERSPECTIVA Y VISIÓN DE LA EDUCACIÓN ESPECIAL (p. 80). Santiago: MINEDUC.

Cornejo, R. (2006). EL EXPERIMENTO E DUCATIVO C HILENO 20 AÑOS DESPUÉS : Una Mirada Crítica a los logros y falencias del sistema escolar. REICE, 4(1).

Decreto 170. (2009). Fija normas para determinar los alumnos con necesidades educativas especiales que serán beneficiarios de las subvenciones para educación especial. Consultado en Abril, 25, 2011 en http://www.mineduc.cl/ biblio/documento/201005031126500.DEC200900170.pdf.

DIPRES. (2009). Minuta Ejecutiva del Programa de Educación Especial Diferencial. Santiago de Chile: Dirección de Presupuestos.

Field, A. (2009). Discovering Statistics Using SPSS. London: SAGE.

Flaspohler, P. D., Elfstrom, J. L., Vanderzee, K. L., Sink, H. E., \& Birchmeier, Z. (2009). Stand by me: the effects of peer and teacher support in mitigating the impact of bullying on quality of life, 46(7), 636-649. doi:10.1002/pits 
García Canclini, N. (1990). Culturas híbridas. Estrategias para entrar y salir de la modernidad. Ciudad de México: Grijalbo.

Godoy, P., Meza, M., \& Salazar, A. (2004). Antecedentes históricos, presente y futuro de la educación especial en Chile. Santiago de Chile: Ministerio de Educación. Extraído el 12 de abril de 2010 desde http://scholar.google.com/ scholar?hl=en\&btnG=Search\&q=intitle:ANTECEDENTES+HISTÓRICOS ,+PRESENTE + Y + FUTURO+DE + LA+EDUCACIÓN+ESPECIAL + EN $+\mathrm{C}$ HILE\#0

Khoury-Kassabri, M. (2006). Student victimization by educational staff in Israel. Child Abuse \& Neglect, 30(6), 691-707. doi:10.1016/j.chiabu.2005.12.003

Khoury-Kassabri, M. (2009). The relationship between staff maltreatment of students and bully-victim group membership. Child Abuse \& Neglect, 33(12), 914-23. doi:10.1016/j.chiabu.2009.05.005

López, V., Bilbao, M., \& Rodríguez, J. (2011). La sala de clases sí importa: Incidencia del clima de aula sobre la percepción de intimidación y victimización entre escolares. Universitas Psychologica, 11(1), 91-102. Extraído el 13 de Marzo de 2013 desde http://revistas.javeriana.edu.co/index.php/revPsycho/ article/view/1002

López, V., Ascorra, P., Bilbao, M., Carrasco, C., Morales, M., Villalobos, B., \& Ayala, Á. (2013). Monitorear la convivencia escolar para fortalecer (no disminuir ) las capacidades de las escuelas. Revista Iberoamericana de Evaluación Educativa, 6(2), 201-219.

López, V., Julio, C., Morales, M., Rojas, C., \& Pérez, M. V. (2014). Barreras culturales para la inclusión: políticas y prácticas de integración en Chile. Revista de Educación, 363(1), 256-281. doi:10.4438/1988-592X-RE-2012-363-180.

Lucas-Molina, B., Williamson, A. A., Pulido, R., \& Pérez-Albéniz, A. (2015). Effects of teacher-student relationships on peer harassment: A multilevel study. Psychology in the Schools, 52(3), 298-315

Marfán, J., Castillo, P., González, R., \& Ferreira, I. (2013). Informe Final de Estudio: Análisis de la implementación de los programas de integración escolar (PIE) en establecimientos que han incorporado estudiantes con necesidades educativas especiales transitorias (NEET). Santiago de Chile: Ministerio de Educación.

MINEDUC. (2012). Encuesta nacional prevención, agresión y acoso escolar $8^{\circ}$ Básico SIMCE 2011. Extraído el 10 de abril de 2013 desde: http://www.mineduc. cl/usuarios/convivencia_escolar/File/Septiembre2012/201207301558020_ Encuesta_nacional_prevencion_agresion_acosoescolar_2011.pdf

OPECH. (2014). Reconstruir la educación pública: ¿Un desafío posible? In Seminario: Análisis de Políticas Educativas.

Peña, M. (2013). Análisis crítico de discurso del Decreto 170 de subvención diferenciada para necesidades educativas especiales: El diagnóstico como herramienta de gestión. Psicoperspectivas. Individuo Y Sociedad, 12, 93-103. doi:10.5027/PSICOPERSPECTIVAS-VOL12-ISSUE2-FULLTEXT-252

Rose, C. A., Monda-Amaya, L. E., \& Espelage, D. L. (2010). Bullying perpetra- 
tion and victimization in special education: A review of the literature. Remedial and Special Education, 32(2), 114-130.

SEGPRES. (2014). Rindiendo cuenta: Balance de cuatro años de Gobierno del Presidente Sebastián Piñera. Santiago.

Shields, N., Nadasen, K., \& Hanneke, C. (2015). Teacher responses to school violence in Cape Town, South Africa. Journal of Applied Social Science, 9(1), 47-64.

Soldatic, K., \& Meekosha, H. (2012). Disability and neoliberal state formations. In Routledge Handbook of Disability Studies (pp. 195-210).

Unicef. (2001). Inclusión de niños con discapacidad en la escuela regular. Extraído el 12 de Enero de 2010 desde: http://www.mineduc.cl/usuarios/edu. especial/doc/201304151207310.doc_Inclusion_Unicef.pdf

Villalobos-Parada, B. (2014). Simulacro de Inclusión: normalización y convivencia escolar. Novedades Educativas, 286 (Octubre).

Villalobos-Parada, B., Carrasco, C., López Leiva, V., Bilbao, M. Á., Morales Pinochet, M., Ortiz Mallegas, S., \& Ayala, Á. (2014). Inclusión y violencia: prevalencia de victimización entre pares en estudiantes referidos a Proyectos de Integración Escolar. Revista Latinoamericana En Educación Inclusiva, $8(2), 161-178$. 